\title{
LncRNA-GAS5 promotes spinal cord repair and the inhibition of neuronal apoptosis via the transplantation of 3D printed scaffold loaded with induced pluripotent stem cell-derived neural stem cells
}

\author{
Rongxue Shao ${ }^{1 \#}$, Changming Li $^{2 \#}$, Yan Chen ${ }^{1}$, Liang Zhang ${ }^{1}$, Hejie Yang ${ }^{1}$, Zhijing Zhang ${ }^{1}$, Jun Yue ${ }^{1}$, \\ Wenshuo Gao ${ }^{3}$, Hang Zhu', Hao Pan ${ }^{1}$, Hui Zhou', Renfu Quan ${ }^{2}$ \\ ${ }^{1}$ Department of Orthopedics, Hangzhou TCM Hospital Affiliated to Zhejiang Chinese Medical University, Hangzhou, China; ${ }^{2}$ Department of \\ Orthopedics, Jiangnan Hospital Affiliated to Zhejiang Chinese Medical University, Hangzhou, China; ${ }^{3}$ Orthopaedic Institute, Zhejiang Chinese \\ Medical University, Hangzhou, China \\ Contributions: (I) Conception and design: R Shao; (II) Administrative support: J Yue, H Zhu, H Pan, H Zhou, R Quan; (III) Provision of study \\ materials or patients: L Zhang, H Yang, Z Zhang; (IV) Collection and assembly of data: Y Chen, W Gao; (V) Data analysis and interpretation: C Li; \\ (VI) Manuscript writing: All authors; (VII) Final approval of manuscript: All authors. \\ \#These authors contributed equally to this work. \\ Correspondence to: Rongxue Shao. Department of Orthopedics, Hangzhou TCM Hospital Affiliated to Zhejiang Chinese Medical University, No. \\ 453, Tiyuchang Road, Hangzhou 310007, China. Email: shaorongxue@163.com; Changming Li. Department of Orthopedics, Jiangnan Hospital \\ Affiliated to Zhejiang Chinese Medical University, No. 152, Yucai Road, Hangzhou 311200, China. Email: 1cmmail@126.com.
}

Background: Stem cell transplantation has been increasingly used for spinal cord repair, and some achievements have been made. However, limited stem cell sources as well as immune rejection and ethical issues have restricted its wide application. Therefore, to achieve further breakthroughs regarding the application of stem cell transplantation to treat spinal cord injury (SCI), it is important to develop a stem cell line that can effectively avoid immune rejection and ethical issues.

Methods: Urine cells (UCs) were induced to differentiate into induced pluripotent stem cells (iPSCs), which then further differentiated into neural stem cells (NSCs). Relevant tests were performed, and threedimensional (3D) printed scaffolds were prepared. Thirty C57BL/6 mice were divided into 5 groups based on a random number table: a sham group, an SCI group, an SCI + control group, an SCI + siNC group, and an SCI + siGAS5 group $(n=6)$. The latter 4 groups replicated SCI models. Mice in the SCI + control group were transplanted with 3D scaffolds loaded with iPSC-derived NSCs (iPSd-NSCs). Mice in the SCI + siNC group and the SCI + siGAS5 group were transplanted with scaffolds loaded with iPSd-NSCs-siNC and 3D scaffolds loaded with iPSd-NSCs-siGAS5, respectively. Mice in the other groups were injected with the same amount of normal saline. Hematoxylin-eosin staining was used to observe the histopathology of the injured spinal cord, the Basso-Mouse Scale was used to assess the motor function of the hind limbs of the mice, and Western blot was used to detect the expression of apoptosis-related proteins after SCI.

Results: iPSd-NSCs were successfully induced and differentiated, and 3D printed heparin sulfate-collagen scaffolds were prepared, inside which a 3D loose porous structure was shown by electron microscopy. Morphological observations showed that iPSd-NSC transplantation improved SCI in mice, while GAS5 silencing inhibited the reparative effect of iPSd-NSC transplantation on SCI in mice. Western blot results indicated that iPSd-NSC transplantation significantly increased the expression level of B cell lymphoma/ leukemia-2 $(\mathrm{Bcl}-2)(\mathrm{P}<0.01)$ but decreased the expression levels of $\mathrm{Bcl}-2$ associated $\mathrm{X}$ protein, cytochrome $\mathrm{C}$, and cleaved caspase- $3(\mathrm{P}<0.001)$.

Conclusions: The overexpression of lncRNA-GAS5 can promote spinal cord repair and inhibit neural apoptosis via the transplantation of $3 \mathrm{D}$ printed scaffolds loaded with iPSd-NSCs. 
Keywords: Long non-coding RNA-GAS5; 3D printed scaffold; iPSC-derived NSCs; spinal cord injury (SCI); neuronal apoptosis

Submitted Apr 08, 2021. Accepted for publication Jun 03, 2021.

doi: $10.21037 / \mathrm{atm}-21-2570$

View this article at: http://dx.doi.org/10.21037/atm-21-2570

\section{Introduction}

Spinal cord injury (SCI) causes different degrees of secondary injury responses, including axonal disconnection, neuronal apoptosis, and necrosis. The toxins released by the lysis of dead cells cause neuronal apoptosis, spinal cord tissue necrosis, cavitation, glial scar hyperplasia, and axonal fragmentation and demyelination near the injury plane, while inhibiting the regeneration of nerve cells and axons (1). SCI treatment has been a challenge in the medical community. In recent years, the emergence of stem cell transplantation technology has provided a new direction for the treatment of SCI. Studies have shown that the transplantation of human umbilical cord mesenchymal stem cells has a reparative effect on SCI, with notable achievements (2). Progress has been made in SCI repair via transplantation of neural stem cells (NSCs). Transplanted NSCs can secrete various neurotrophic factors, which can improve the local microenvironment and promote the regeneration of endogenous NSCs in injured spinal cord, facilitating the regeneration of injured nerve axons that can then be passed through the injured cavity in the spinal cord, thereby promoting the repair and functional reconstruction of the injured spinal cord (3). However, limited stem cell sources, ethical issues and immune rejection have restricted the development of stem cell transplantation. LncRNAGAS5, located on chromosome 1 of the human genome, is a growth suppressor, plays an important regulatory function in the regulation of biological processes, such as cell proliferation, apoptosis, migration, and invasion, and is currently considered to be associated with various biological behaviors, such as tumor growth, vascular remodeling, and inflammatory response (4).

In this study, induced pluripotent stem cells (iPSCs) derived from urine cells (UCs) were induced to differentiate into NSCs, and three-dimensional (3D) printed scaffolds loaded with iPSC-derived NSCs (iPSd-NSCs) were modified using long non-coding RNA-growth arrestspecific transcript 5 (lncRNA-GAS5) and transplanted into injured spinal cord to further investigate the role of lncRNA-GAS5 in the repair of SCI in mice.
We present the following article in accordance with the ARRIVE reporting checklist (available at http://dx.doi. org/10.21037/atm-21-2570).

\section{Methods}

\section{Experimental materials}

Thirty C57BL/6 mice (8-12 weeks old) were purchased from Zhejiang Chinese Medical University. All experimental procedures were conducted at the Experimental Animal Management Center of Jiangnan Hospital Affiliated with Zhejiang Chinese Medical University. Experiments were performed under a project license (No.: 20170625182365) granted by ethics board of Jiangnan Hospital Affiliated with Zhejiang Chinese Medical University, in compliance with national guidelines for the care and use of animals. A protocol was prepared before the study without registration. StemPro cell dissociation reagent (A11105, Sigma, USA), neurobasal medium (21103049, Sigma, USA), advanced DMEM/F12 (12634010, Sigma, USA), Alexa Fluor 594 (1:200 dilution, Sigma, USA), pcDNA3.1 vector (V79020, Sigma, USA), siRNA GAS5 (siGAS5; siB1356203228-1-5, Bioss, China), Lipofectamine ${ }^{\circledR} 2000$ transfection reagent (11668, Sigma, USA), polylactic-co-glycolic acid (P133293, Aladdin), glial fibrillary acidic protein (GFAP) (ab53554, Sigma, USA), tubulin ( $\beta$-tubulin III, ab52623, Sigma, USA), growth-associated protein 43 (GAP-43) (ab75810, Sigma, USA), nestin (OM264981, Sigma, USA), fetal bovine serum (F8687, Sigma, USA), ALP detection kits (KLALP-Ra, Shanghai Kanglang Biological Technology Co., Ltd., China), heparin sulfate (QC30767, GlpBio, USA), a cryostat (CM3050 S, Leica Microsystems, Japan), a 3D printer (HangZhou Regenovo Biotechnology., Ltd., China), H\&E staining kits (G1120, Solorbio, China), and TUNEL apoptosis detection kits $(20 \mathrm{mg} / \mathrm{ml}$, ST533, China) were used in the study.

\section{Differentiation and identification of iPSd-NSCs}

The isolation of UCs and the construction of iPSCs 
followed the methods described by Zhou et al. (5). iPSCs were passaged after successful induction and differentiation. Feeder cells were seeded 1 day before selecting the clones. Under a microscope, monoclonal cells with good morphology were selected for alkaline phosphatase (ALP) activity analysis (ALP staining) using an ALP detection kit. A total of $1 \times 10^{6}$ iPSCs were injected subcutaneously into the back of 5-week-old male SD mice, and the formation of teratomas was verified by hematoxylin-eosin (HE) staining. iPSCs were fixed with $4 \%$ paraformaldehyde for $15 \mathrm{~min}$ at room temperature, permeabilized with $0.25 \%$ (V/V) polyethylene glycol octyl phenyl ether (Triton $\mathrm{X}-100) /$ phosphate-buffered saline (PBS) for $10 \mathrm{~min}$, and blocked with $4 \%$ (W/V) bovine serum albumin for $1 \mathrm{~h}$ at room temperature. Then, the iPSCs were incubated with primary antibodies at $4{ }^{\circ} \mathrm{C}$ overnight and then incubated with secondary antibodies for $30 \mathrm{~min}$ at room temperature. Nuclei were stained with 4',6-diamidino-2-phenylindole (DAPI). The primary antibodies used were anti-OCT4 (1:350, rabbit polyclonal antibody), anti-NANOG (1:1,000, rabbit monoclonal antibody), anti-TRA-1-81 (1:100, mouse monoclonal antibody), and anti-TRA-1-60 (1:100, mouse monoclonal antibody). The secondary antibodies were goat anti-rabbit Alexa Fluor 488 or 568 and goat antimouse Alexa Fluor 647. The samples were imaged under a fluorescence microscope.

Digested iPSC colonies were cultured in embryoid body (EB) medium to form mature EBs, induced with retinoic acid + serum-free medium, and then induced and cultured in EB medium for 4 days; the medium was then replaced with serum-free medium for adherent culture. The resulting neurosphere-like clones obtained by screening and culture were named iPSCs-NSCs. The induced and differentiated iPSCs-NSCs were cultured in a medium containing $10 \%$ fetal bovine serum and were then loaded with $0.1 \%$ gelatin to detect $\beta$-tubulin III and GFAP expression using immunofluorescence. Cells were also grown in culture plates and were fixed with $4 \%$ paraformaldehyde for $15 \mathrm{~min}$, after which $5 \%$ bovine serum albumin was added dropwise for blocking for $30 \mathrm{~min}$ at $37^{\circ} \mathrm{C}$. The blocking solution was removed using a pipette, and the primary antibody (antinestin, 1:200) was added dropwise to the culture plate, and the cells were incubated overnight at $4{ }^{\circ} \mathrm{C}$. The cells were rinsed with $\mathrm{PBS}$, and residual $\mathrm{PBS}$ in the culture plate was removed using a pipette. Next, FITC-conjugated secondary antibody (1:100) was added dropwise to the culture plate, and the cells were incubated at $37^{\circ} \mathrm{C}$ for $45 \mathrm{~min}$. The cells were rinsed with PBS, and DAPI was added dropwise to the culture plate, followed by incubation in the dark for 5 min to stain nuclei. Excess DAPI was removed using PBS, and the cells was sealed with $50 \%$ glycerol. The expression of nestin, a specific marker of iPSCs-NSCs, was observed under a fluorescence microscope.

\section{Preparation and pretreatment of $3 D$ heparin sulfate- collagen scaffolds}

One milligram of heparin sulfate, $100 \mathrm{mg}$ of type I collagen, and $10 \mathrm{mg}$ of type II collagen were mixed with $200 \mathrm{mg}$ of polylactic-co-glycolic acid, and the thickener improved the viscosity and stability of the slurry. Under a nitrogen stream, the prepared slurry was transferred to a material bucket, and the partial pressure was set. The $3 \mathrm{D}$ printer nozzle was selected, and the material out-feed was calibrated. The printing parameters were set and adjusted, including material out-feed temperature, platform temperature, etc. Once the three-dimensional scaffolds were generated, they were kept at room temperature for more than $4 \mathrm{~h}$ and continuously dried in a thermostatic drying oven at 45 and $60{ }^{\circ} \mathrm{C}$ for $12 \mathrm{~h}$ each. The morphology of the scaffolds was observed under an electron microscope.

Then, each 3D scaffold was trimmed into small segments (approximately $2 \mathrm{~mm}^{2}$ ) and soaked in stem cell medium for $48 \mathrm{~h}$. The scaffolds were then air-dried in a laminar flow hood and sterilized prior to use. On the $7^{\text {th }}$ day of culture, iPSCs-NSCs cells were digested and counted, and $1 \times 10^{6}$ cells were concentrated to $0.2 \mathrm{~mL}$ and dropped onto the pretreated scaffolds, which were placed in an incubator for $2 \mathrm{~h}$ before being used for the experiment.

\section{Grouping and intervention}

Thirty C57BL/6 mice were divided into 5 groups based on a random number table: a sham group, an SCI group, an SCI+control group, an SCI+siNC group and an $\mathrm{SCI}+$ siGAS5 group ( $\mathrm{n}=6$ mice). In the sham group, only the lamina was removed, and the spinal cord was not injured. In the remaining groups, the modified Allen method was used to reproduce SCI. After the model was successfully established, the animals were reared for 7 days before the experiment. In the SCI+control group, a 3D scaffold loaded with $5 \times 10^{5}$ iPSd-NSCs was implanted into the center of the lesion in each mouse; in the SCI+siNC group and the SCI+siGAS5 group, 3D scaffolds loaded with the same amount of iPSd-NSCs-siNC and iPSd-NSCs-siGAS5, respectively, were implanted using the above method; and 
in the sham group and the SCI group, 3D scaffolds without any cell coating were implanted.

\section{Assays and procedures}

\section{Transfection of iPSd-NSCs}

Full-length mouse GAS5 cDNA was amplified and then cloned into the pcDNA3.1 vector to construct the GAS5 plasmid. The empty pcDNA3.1 vector was used as a negative control (NC). siGAS5 and its corresponding NC (siNC) were obtained. iPSCs were seeded in $60-\mathrm{mm}$ dishes and cultured to $70-90 \%$ confluency, after which they were transfected with siRNA and the plasmids using Lipofectamine $^{\circledR} 2000$. After 48 h of transfection, cells from each group were used for the subsequent experiments.

\section{Methyl thiazolyl tetrazolium (MTT) assay}

iPSd-NSCs transfected with GAS5 were seeded into polystyrene tissue culture plates (96-well) $\left(1 \times 10^{5}\right.$ cells/well), and the medium was replaced with $100 \mu \mathrm{L}$ of MTT solution on the $1^{\text {st }}, 3^{\text {rd }}$, and $5^{\text {th }}$ days of seeding. After incubation for $4 \mathrm{~h}$ at $37^{\circ} \mathrm{C}$ in $5 \% \mathrm{CO}_{2}$, the supernatant was removed, and the MTT solution was replaced with $100 \mu \mathrm{L}$ of dimethyl sulfoxide to dissolve the product (methoxypyrimidine). The optical density at $570 \mathrm{~nm}$ was read with a microplate reader.

\section{Rating of hindlimb motor function}

The hindlimb motor function of mice was assessed using the 9-point Basso-Mouse Scale (BMS). The BMS scores for each group were recorded on the $1^{\text {st }}, 7^{\text {th }}, 14^{\text {th }}, 21^{\text {st }}$, and $28^{\text {th }}$ days after SCI, and differences were compared.

\section{Histomorphological observation}

Histopathological examination of the spinal cord was performed via HE staining. The above experimental mice were anesthetized by intraperitoneal injection of $10 \%$ chloral hydrate $(0.3 \mathrm{~mL} / 100 \mathrm{~g})$. A longitudinal incision was made along the middle of the chest. The tissue was cut layer by layer, and the ribs were cut to expose the heart. The left ventricle and the right atrium were incised, and cannulation was performed from the left ventricle along the direction of the aorta. Infusion of normal saline was rapidly performed until the perfusate returning to the right atrium was clear. Then, $4 \%$ paraformaldehyde PD solution $(\mathrm{pH} 7.4)$ was rapidly infused at a flow rate of $50 \mathrm{ml} / \mathrm{min}$ for 3-4 $\mathrm{min}$, followed by $10 \mathrm{~mL} / \mathrm{min}$ for $20 \mathrm{~min}$. Ten millimeters of spinal cord corresponding to the T9-T10 vertebral bodies was taken and washed with normal saline, and the specimens were fixed in $10 \%$ formaldehyde for $24-48 \mathrm{~h}$ and routinely embedded in paraffin. The specimens were dehydrated in an ethanol series, cleared in xylene, embedded in paraffin, and sliced into 5 - $\mu \mathrm{m}$ sections. HE staining was performed, and spinal cord tissue from each group was observed under a microscope to observe pathological changes.

\section{Western blot analysis}

Spinal cord samples $(50-100 \mu \mathrm{g})$ were mixed with buffer solution $(5 \times$ volume) and boiled for $10 \mathrm{~min}$. Proteins were resolved on a sodium dodecyl sulfate -polyacrylamide gel and transferred to a polyvinylidene fluoride membrane. The membrane was incubated with primary antibody [rabbit antiBcl-2 antibody (1:40, dilution, Bioworld, China), rabbit antiBax antibody (1:20, dilution, Bioworld, China), rabbit antiCyt-C antibody (1:40, dilution, Bioworld, China), and rabbit anti-caspase-3 antibody (1:40, dilution, Bioworld, China)] at $4{ }^{\circ} \mathrm{C}$ overnight and then incubated with the corresponding secondary antibody [goat anti-rabbit IgG (H \& L chains)]. The expression levels of B cell lymphoma/leukemia-2 (Bcl-2), Bcl-2 associated $\mathrm{X}$ protein (Bax), cytochrome $\mathrm{C}$, and cleaved caspase-3 were assessed. Quantity One image analysis software was used to analyze the optical density, and the ratio of the optical density of the target band to that of the band corresponding to the internal control protein $\beta$-actin was the relative expression value of the target protein.

\section{Statistical analysis}

All data were analyzed using SPSS 19.0. The data are presented as mean $\pm \mathrm{SD}(\bar{x} \pm s)$. When comparing groups, if the data had a normal distribution and the variance was homogeneous, then one-way ANOVA (least significant difference) and the Student-Newman-Keuls test were used for the analysis. When the variance was not homogeneous, the Tamhane T2 and Dunnett T3 methods were used for variance tests and pairwise comparisons. $\mathrm{P}<0.05$ was considered statistically significant.

\section{Results}

\section{Characterization of iPSd-NSCs}

Validation experiments indicated that UC-derived iPSCs were pluripotent and expressed the pluripotent proteins OCT4, NANOG, TRA-1-81, and TRA-1-60 (Figure 1). The teratoma test confirmed that they had the ability to differentiate into 3 germ layers: intestinal epithelium 

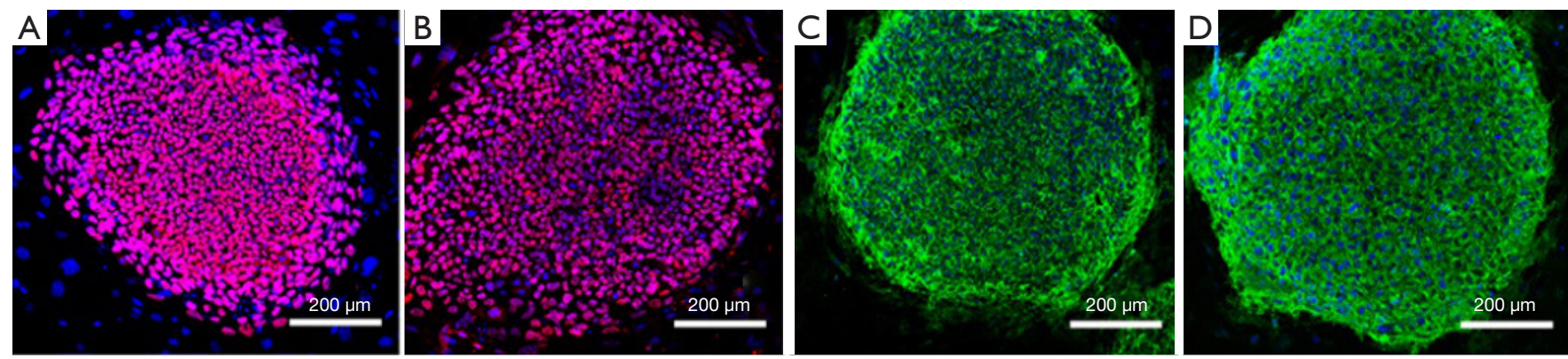

Figure 1 Immunofluorescence staining of pluripotent proteins expressed in induced pluripotent stem cells (iPSCs) [A, expression of the pluripotent protein OCT4 (1:350, rabbit polyclonal); (B) expression of the pluripotent protein NANOG (1:1,000, rabbit monoclonal antibody); (C) expression of the pluripotent protein TRA-1-81 (1:100, mouse monoclonal antibody); (D) expression of the pluripotent protein TRA-1-60 (1:100, mouse monoclonal antibody); ×200].
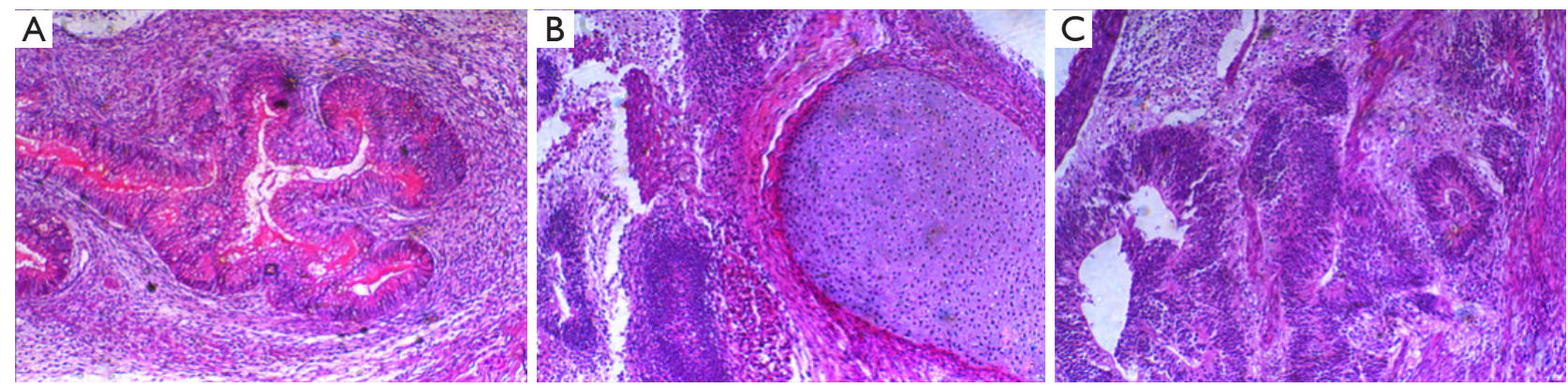

Figure $2 \mathrm{HE}$ staining of teratoma tissue sections [A, intestinal epithelium (endoderm); B, cartilage (mesoderm); C, neuroepithelium (ectoderm); HE staining, ×200].
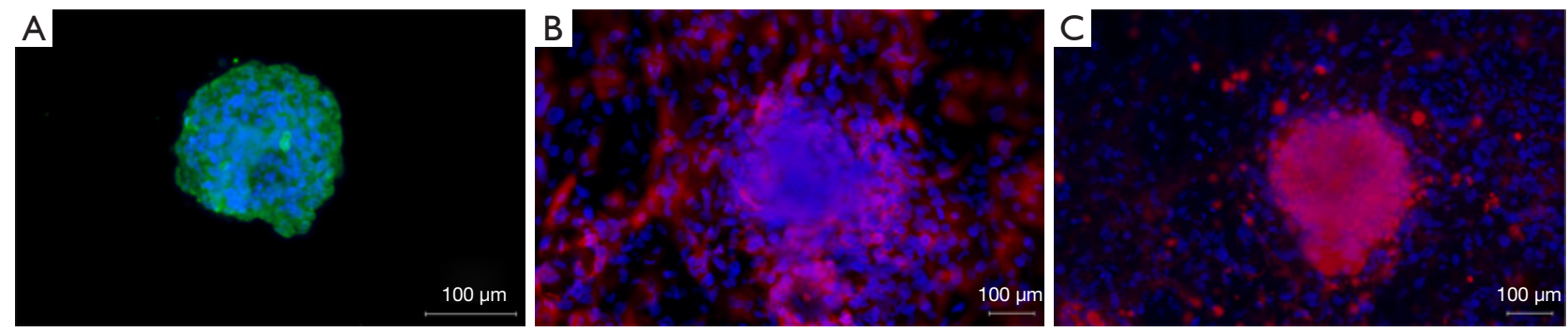

Figure 3 Immunofluorescence characterization of iPSCs-NSCs [A, the expression of nestin (1:200, rabbit polyclonal), a characteristic marker of induced pluripotent stem cells (iPSCs)-neural stem cells (NSCs); B, the expression of tubulin ( $\beta$-tubulin III) (1:200, mouse monoclonal antibody) in iPSCs-NSCs; C, the expression of glial fibrillary acidic protein (GFAP) (1:200, mouse monoclonal antibody) in iPSCs-NSCs; $\times 100]$. iPSC, induced pluripotent stem cell; NSCs, neural stem cell.

(endoderm), cartilage (mesoderm), and neuroepithelium (ectoderm) (Figure 2). In immunofluorescence images, the target protein nestin in iPSCs-NSCs exhibited green fluorescence, and nuclei exhibited blue fluorescence after DAPI staining. When combined with the abovementioned cell morphology analysis, iPSCs-NSCs could be identified (Figure 3A). Additionally, in immunofluorescence images, the target protein $\beta$-tubulin exhibited red fluorescence, and nuclei exhibited blue fluorescence after DAPI staining. The cells showed high levels of expression and 

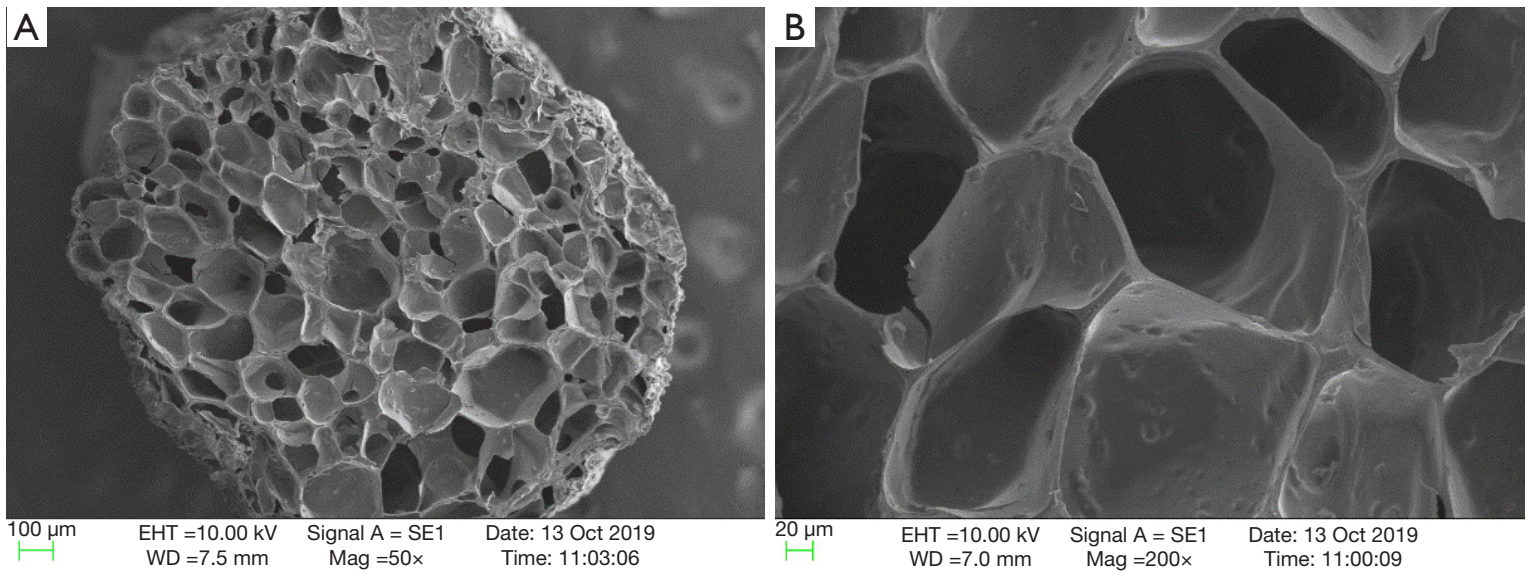

Figure 4 Scanning electron microscopy showed that the cross-section of the scaffold was loose and porous (left $\times 50$, right $\times 200)$.
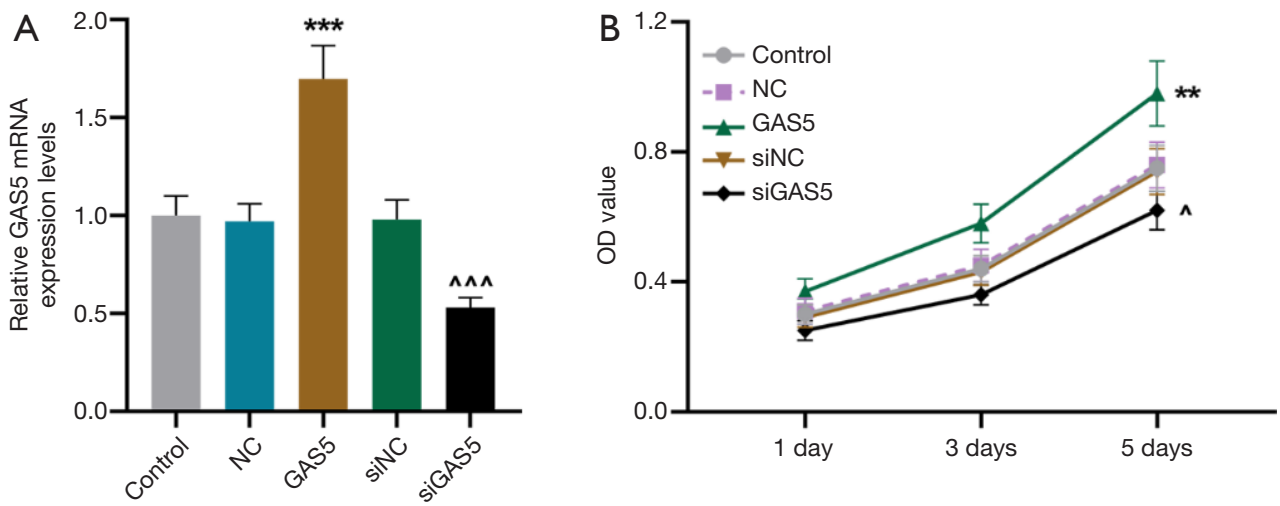

Figure 5 The expression of GAS5 (A) and the activity of iPSd-NSCs at different time points (B). Compared with Control group, **, P<0.01; ***, $\mathrm{P}<0.001 ; \wedge, \mathrm{P}<0.05 ; \wedge \wedge \wedge, \mathrm{P}<0.001$. iPSC, induced pluripotent stem cell; NSCs, neural stem cell; NC, negative control.

had good differentiation ability (Figure 3B). Last, in immunofluorescence images, the target protein GFAP exhibited red fluorescence, and nuclei exhibited blue fluorescence after DAPI staining. The cells had peripheral extension and good differentiation ability (Figure 3C).

\section{Characterization of $3 D$ printed scaffolds}

The 3D printed heparin sulfate-collagen scaffolds had a three-dimensional porous structure. Scanning electron microscopy indicated that the cross-section of the scaffold was loose and porous, with a pore size between 50 and $200 \mu \mathrm{m}$ (Figure 4).

\section{Regulation of iPSd-NSC activity by GASS}

The SCI+control (control) group was transplanted with a $3 \mathrm{D}$ scaffold loaded with $5 \times 10^{5}$ iPSd-NSCs, the GAS5 group was transplanted with a $3 \mathrm{D}$ scaffold loaded with the same amount of iPSd-NSCs-GAS5, and the NC group was transplanted with a 3D scaffold loaded with iPSd-NSCsNC. The GAS5 plasmid significantly increased the level of GAS5 5 in iPSd-NSCs, while siGAS5 decreased the level of GAS5 in iPSd-NSCs (Figure $5 A, \mathrm{P}<0.001$ ). The results from the MTT assay indicated that the GAS5 overexpression significantly increased the activity of iPSd-NSCs at day 5 $(\mathrm{P}<0.01)$, while siGAS5 significantly inhibited the activity of iPSd-NSCs $(\mathrm{P}<0.05)$ (Figure 5B).

\section{Histomorphological observations}

Healthy spinal cord tissue was observed in the sham group. In the SCI group, hemorrhage, liquefaction, and inflammatory cell infiltration were observed at the SCI 

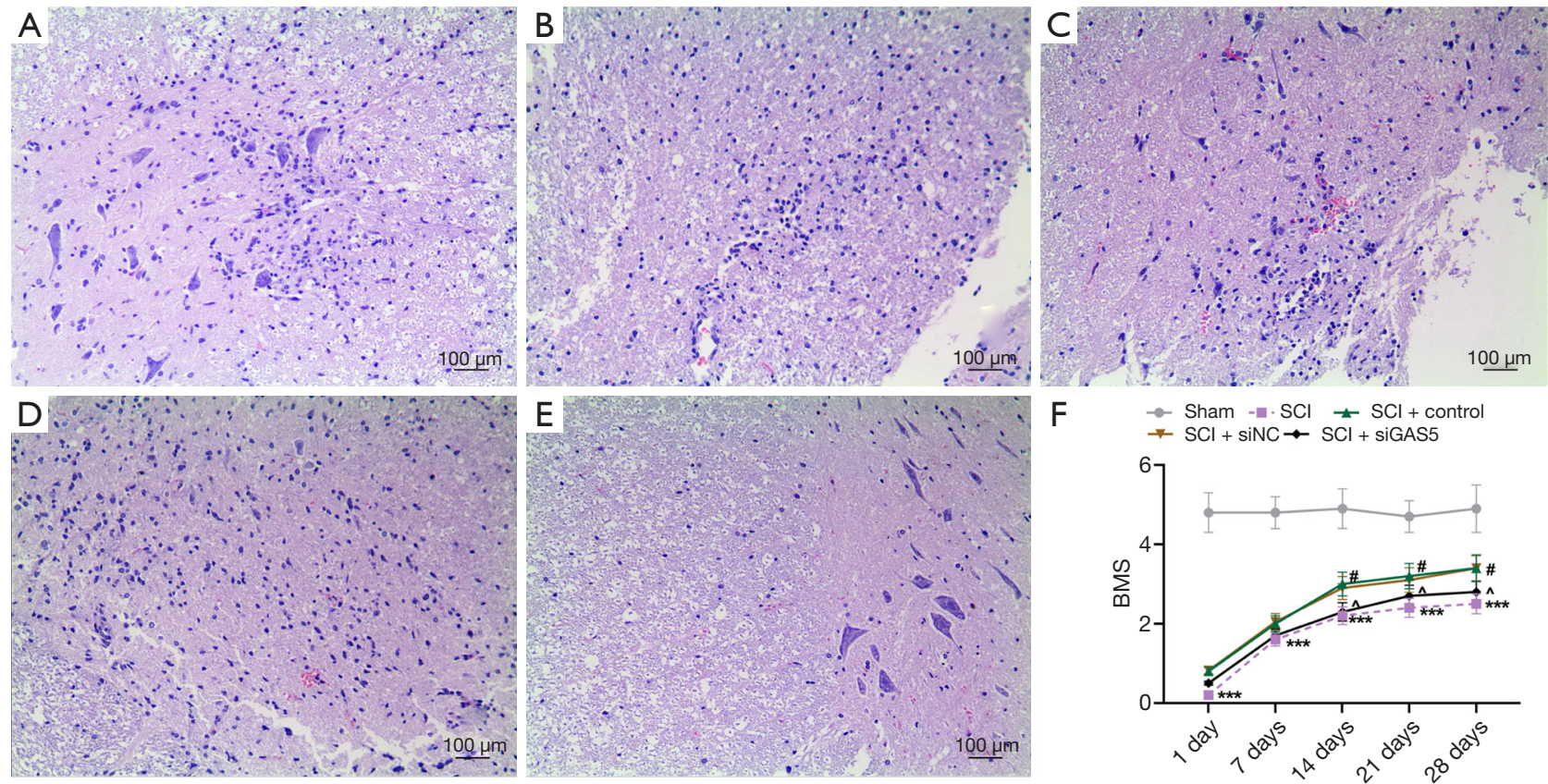

Figure 6 Morphological changes in spinal cord tissue from mice in each group (A,B,C,D,E; HE staining) and BMS scores for mice in each group at different time points $(\mathrm{F}) .^{* * *}, \mathrm{P}<0.001 ;{ }^{\wedge}, \mathrm{P}<0.05$; ${ }^{*}, \mathrm{P}<0.05$. BMS, Basso-Mouse Scale; SCI, spinal cord injury.

site, the boundary between the gray matter and white matter of the spinal cord was unclear, and the cytoplasm of neurons showed granular and vacuolar degeneration. In the SCI+control group and the SCI+siNC group, there was less bleeding from the SCI, less inflammatory cell infiltration, and a smaller cavity area in the cross-section of the spinal cord. In the SCI+siGAS5 group, there was local hemorrhage, significant inflammatory cell infiltration, and cytoplasmic vacuolar degeneration of the neurons at the SCI site (Figure 6A,B,C,D,E).

\section{Regulatory effects of IncRNA-GAS5 on SCI repair and neuronal apoptosis inbibition via the transplantation of 3D scaffolds loaded with iPSd-NSCs}

The mean BMS score in the SCI group was significantly lower than that in the sham group at different time points after SCI $(\mathrm{P}<0.001)$. On the $14^{\text {th }}, 21^{\text {st }}$, and $28^{\text {th }}$ days, the mean BMS scores in the SCI+control group were significantly higher than those in the SCI group $(\mathrm{P}<0.05)$, and the mean BMS scores in the SCI+siGAS5 group were significantly lower than those in the SCI+siNC group $(\mathrm{P}<0.05)$ (Figure 6F).

In addition, iPSd-NSC transplantation markedly reduced neuronal apoptosis in the spinal cord, while GAS5 silencing partially abolished this effect. Western blot results indicated that iPSd-NSC transplantation significantly increased the expression of $\mathrm{Bcl}-2$ and reduced the expression of Bax, cytochrome $\mathrm{C}$ and cleaved caspase- 3 . The ratio of Bcl2 to Bax in sham, SCI, SCI+control, SCI+siNC and SCI+siGAS5 groups were 1.98, 0.1, 1.01, 1.23 and 1.675, respectively (Figure 7).

\section{Discussion}

The repair and functional reconstruction of SCI has been a hot topic in the medical community. With the development of tissue engineering, biological scaffold-loaded seed cell transplantation therapy has been increasingly used to repair spinal cords because of the similarities in the dynamic interactions between cells and the extracellular matrix in normal tissues in vivo $(6,7)$. Studies have shown that $3 \mathrm{D}$ printed scaffolds loaded with NeuroD1-modified NSCs can promote the reconstruction of neural circuits and the recovery of motor and sensory function in rats with SCI (8). Additionally, collagen-heparin sulfate scaffolds combined with NSCs can promote the regeneration of nerve fibers at SCI sites and improve the motor function of hind limbs in rats (9). However, NSCs can only be obtained from limited sources, are mired in ethical controversies, and are associated 

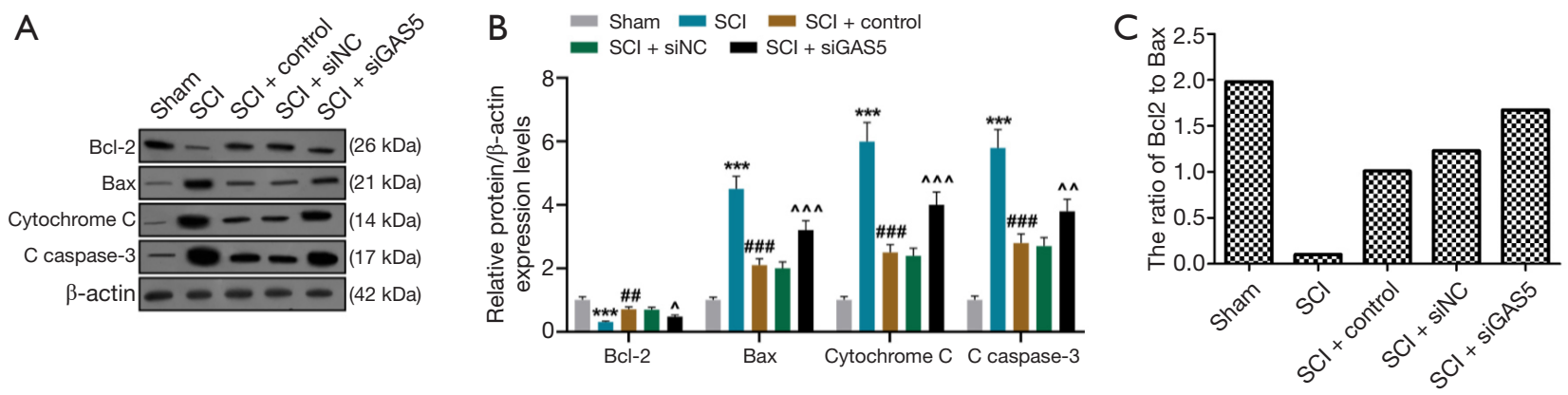

Figure 7 Western blot results for Bcl-2, Bax, cytochrome C and cleaved caspase-3 in each group (A). iPSd-NSC transplantation significantly increased the expression of Bcl-2 and reduced the expression of Bax, cytochrome $\mathrm{C}$ and cleaved caspase-3. Compared with Sham group, ***, $\mathrm{P}<0.001 ;{ }^{\# \#}, \mathrm{P}<0.01 ;{ }^{\# \# \#}, \mathrm{P}<0.001 ; \wedge, \mathrm{P}<0.05 ; \wedge \wedge, \mathrm{P}<0.01 ; \wedge \wedge, \mathrm{P}<0.001$. (B). The ratio of Bcl2 to Bax was calculated and typed (C). SCI, spinal cord injury.

with immune rejection. The iPSCs used in this study were obtained by reprogramming UCs from a homologous host. The abovementioned iPSCs differentiated into NSCs after induction. The characterization results indicated that the differentiated cells aggregated into neurospheres and that the NSC marker nestin was expressed. The results showed that iPSCs successfully differentiated into NSCs. IPSCs are multipotent stem cells established by reprogramming somatic cells, which are not associated with ethical and immune rejection issues. Moreover, under different induction conditions, iPSCs can be transformed into a variety of cells. iPSCs have successfully differentiated into cells of the nervous system (10), hematopoietic system (11), and other systems (12) under specific culture conditions in vitro.

It participates in the regulation of cell proliferation, ion channels, and cell growth through the following 3 mechanisms: (I) GAS5 binds to regulatory proteins with transcriptional function to regulate the expression of related genes; (II) GAS5 negatively regulates the expression of its downstream target genes by binding to targeted miRNAs via mimicking molecular sponges; and (III) GAS5 is directly involved in the regulation of protein translation (13). In this study, we show that GAS5 overexpression significantly improves the viability of iPSd-NSCs. Studies have shown that GAS5 is essential for the self-renewal and pluripotency of iPSCs. It forms a positive feedback network with a set of key pluripotency modulators (Sox2, Oct4, Nanog, Tcl1, Esrrb, and Tet1). When the GAS5 gene is knocked out, the efficiency of iPSC reprogramming is significantly reduced (14), a finding that is similar to the results of the present study. In addition, the BMS scores indicated that NSC transplantation significantly improved the motor function of the hindlimbs, while GAS5 silencing significantly inhibited the beneficial effect of NSC transplantation on the hindlimb motor function in SCI mice.

After SCI, the expression of related apoptosis factors is modified. Among them, Bcl-2 and Bax are important apoptosis-regulating proteins in acute SCI. Bcl-2 inhibits apoptosis, while Bax promotes apoptosis, and the Bcl-2/ Bax ratio determines whether cells undergo apoptosis, with an increased ratio promoting cell survival and a decreased ratio promoting cell death (15). In addition, cytochrome $\mathrm{C}$ is a key factor in the mitochondria-mediated apoptotic pathway. When cells are damaged, the permeability of the mitochondrial membrane changes, and cytochrome $\mathrm{C}$ and caspase- 3 are released to activate apoptosis through a signaling cascade (16). The results of this study confirmed that the implantation of GAS5-modified iPSd-NSCs into injured spinal cord significantly increased the expression of Bcl-2, decreased the expression of Bax, cytochrome C, and cleaved caspase-3, and partially reversed neuronal apoptosis, thereby promoting spinal cord repair in SCI mice.

In summary, IncRNA-GAS5 promotes the reparative effect of the transplantation of 3D printed scaffolds loaded with iPSd-NSCs after SCI, significantly restoring motor function. These effects may occur because the 3D printed heparin sulfate-collagen scaffolds provide a bridge for interrupted neural circuits and iPSCs-NSCs can improve the damaged spinal cord microenvironment. Additionally, lncRNA-GAS5 has a regulatory effect on the activity of iPSd-NSCs and inhibits neuronal apoptosis, thus further promoting SCI repair. However, the current research is only in the basic experimental stage, and more samples are 
needed for clinical application.

\section{Acknowledgments}

Funding: This work was supported by (I) Natural Science Foundation of Zhejiang Province (No. Y19H060066), (II) Major Programs for Social Development and Technology Development (No. 2017209), and (III) The Medical and Health Science and Technology Plan Project of Zhejiang Province (No. 2018248755 and No. 2019RC074).

\section{Footnote}

Reporting Checklist: The authors have completed the ARRIVE reporting checklist. Available at http://dx.doi. org/10.21037/atm-21-2570

Data Sharing Statement: Available at http://dx.doi. org/10.21037/atm-21-2570

Conflicts of Interest: All authors have completed the ICMJE uniform disclosure form (available at http://dx.doi. org/10.21037/atm-21-2570). The authors have no conflicts of interest to declare.

Ethical Statement: The authors are accountable for all aspects of the work in ensuring that questions related to the accuracy or integrity of any part of the work are appropriately investigated and resolved. Experiments were performed under a project license (No.: 20170625182365) granted by ethics board of Jiangnan Hospital Affiliated with Zhejiang Chinese Medical University, in compliance with national guidelines for the care and use of animals.

Open Access Statement: This is an Open Access article distributed in accordance with the Creative Commons Attribution-NonCommercial-NoDerivs 4.0 International License (CC BY-NC-ND 4.0), which permits the noncommercial replication and distribution of the article with the strict proviso that no changes or edits are made and the original work is properly cited (including links to both the formal publication through the relevant DOI and the license). See: https://creativecommons.org/licenses/by-nc-nd/4.0/.

\section{References}

1. Xu Z, Zhang K, Wang Q, et al. MicroRNA-124 improves functional recovery and suppresses Bax-dependent apoptosis in rats following spinal cord injury. Mol Med Rep 2019;19:2551-60.

2. Yang Y, Pang M, Chen YY, et al. Human umbilical cord mesenchymal stem cells to treat spinal cord injury in the early chronic phase: study protocol for a prospective, multicenter, randomized, placebo-controlled, singleblinded clinical trial. Neural Regen Res 2020;15:1532-8.

3. Jeong SK, Choi I, Jeon SR. Current Status and Future Strategies to Treat Spinal Cord Injury with Adult Stem Cells. J Korean Neurosurg Soc 2020;63:153-62.

4. Xu X, Hou J, Lv J, et al. Overexpression of lncRNA GAS5 suppresses prostatic epithelial cell proliferation by regulating COX-2 in chronic non-bacterial prostatitis. Cell Cycle 2019;18:923-31.

5. Zhou T, Benda C, Duzinger S, et al. Generation of induced pluripotent stem cells from urine. J Am Soc Nephrol 2011;22:1221-8.

6. Deng LX, Deng P, Ruan Y, et al. A novel growthpromoting pathway formed by GDNF-overexpressing Schwann cells promotes propriospinal axonal regeneration, synapse formation, and partial recovery of function after spinal cord injury. J Neurosci 2013;33:5655-67.

7. Shrestha B, Coykendall K, Li Y, et al. Repair of injured spinal cord using biomaterial scaffolds and stem cells. Stem Cell Res Ther 2014;5:91.

8. Zhang R, Li X, Cheng S, et al. Three-dimensional scaffolds seeded with NeuroDl-modified neural stem cells for repair of spinal cord injury. Chinese Journal of Traumatology 2016;32:536-41.

9. Cao Z, Zheng B, Zhong L, et al. Collagen/heparin sulfate scaffold combined with neural stem cells promote motor function recovery after spinal cord injury. Chinese Journal of Tissue Engineering Research 2019;23:5454-61.

10. Perera TH, Lu X, Howell SM, et al. Combination of IKVAV, LRE, and GPQGIWGQ Bioactive Signaling Peptides Increases Human Induced Pluripotent Stem Cell Derived Neural Stem Cells Extracellular Matrix Remodeling and Neurite Extension. Adv Biosyst 2020;4:e2000084.

11. Yan X, Qin H, Qu C, et al. iPS cells reprogrammed from human mesenchymal-like stem/progenitor cells of dental tissue origin. Stem Cells Dev 2010;19:469-80.

12. Murai N, Mitalipova M, Jaenisch R. Functional analysis of CX3CR1 in human induced pluripotent stem (iPS) cell-derived microglia-like cells. Eur J Neurosci 2020;52:3667-78.

13. Gu J, Wang Y, Wang X, et al. Effect of the LncRNA GAS5-MiR-23a-ATG3 Axis in Regulating Autophagy 
in Patients with Breast Cancer. Cell Physiol Biochem 2018;48:194-207.

14. Tu J, Tian G, Cheung HH, et al. Gas5 is an essential lncRNA regulator for self-renewal and pluripotency of mouse embryonic stem cells and induced pluripotent stem cells. Stem Cell Res Ther 2018;9:71.

Cite this article as: Shao R, Li C, Chen Y, Zhang L, Yang H, Zhang Z, Yue J, Gao W, Zhu H, Pan H, Zhou H, Quan R. LncRNA-GAS5 promotes spinal cord repair and the inhibition of neuronal apoptosis via the transplantation of $3 \mathrm{D}$ printed scaffold loaded with induced pluripotent stem cell-derived neural stem cells. Ann Transl Med 2021;9(11):931. doi: 10.21037/ atm-21-2570
15. Ginsberg D. The epidemiology and pathophysiology of neurogenic bladder. Am J Manag Care 2013;19:s191-6.

16. Bleicken S, Wagner C, García-Sáez AJ. Mechanistic differences in the membrane activity of Bax and Bcl-xL correlate with their opposing roles in apoptosis. Biophys J 2013;104:421-31. 